\title{
Estresse e cultura organizacional: o papel do psicólogo na prevenção e tratamento da Síndrome de Burnout
}

\section{Stress and organizational culture: the role of the psychologist in the prevention and treatment of Burnout Syndrome}

Walter Rodrigues Marques ${ }^{1 *}$, Amanda Marcos Coelho1 ${ }^{2}$, Francisca Rodrigues Marques2 ${ }^{3}$, Jonathan Serra Birino3 ${ }^{3}$, Ivameire Martins Alves Araújo4 ${ }^{1}$, Raquel Marques Costa5 $5^{5}$, Verbena

Vieira Ribeiro de Sousa6 ${ }^{6}$, André Nogueira Machado $7^{4}$

\begin{abstract}
RESUMO
O ensaio discute o processo de vivência de prazer-sofrimento do estresse na cultura organizacional. A qualidade de vida nos remete a uma reflexão crítica e sistêmica, em meio ao mundo do trabalho. Tendo em vista que esta vivência traz prejuízos para o colaborador e para a organização (diversos problemas de saúde, absenteísmo e queda de produtividade), objetivamos analisar as implicações da cultura organizacional que favorecem o estresse no colaborador. Utilizamos como metodologia a análise da literatura pertinente à Síndrome de Burnout e das relações do mundo do trabalho assim como os aspectos destacados pelos autores sobre as formas de prevenção e tratamento da síndrome. Destacamos que os referenciais teóricos que utilizamos situam-se nas áreas de saúde, administração e psicologia. Por fim, destacamos que o psicólogo é figura central no processo de prevenção e tratamento à Síndrome de Burnout.
\end{abstract}

Palavras-chave: Estresse; Cultura e clima organizacional; Síndrome de Burnout; Psicologia do trabalho e das organizações; Psicologia.

\section{ABSTRACT}

The essay discusses the process of experiencing pleasure-stress in organizational culture. Quality of life leads us to critical and systemic reflection in the midst of the world of work. Considering that this experience brings harm to the employee and to the organization (several health problems, absenteeism and reduced productivity), we aim to analyze the implications of the organizational culture that favor stress on the employee. We used as a methodology the analysis of literature relevant to Burnout Syndrome and the relationships in the world of work, as well as the aspects highlighted by the authors about the forms of prevention and treatment of the syndrome. We emphasize that the theoretical frameworks we use are in the areas of health, administration and psychology. Finally, we emphasize that the psychologist is a central figure in the process of prevention and treatment of Burnout Syndrome.

Keywords: Stress; Culture and organizational climate; Burnout syndrome; Psychology of work and organizations; Psychology.

\footnotetext{
${ }^{1}$ SEDUC-MA (Secretaria de Estado da Educação do Maranhão)

*E-mail: walter.marques@ @rof.edu.ma.gov.br

2 UEMA (Universidade Estadual do Maranhão)

${ }^{3}$ IESF (Instituto Superior Franciscano)

${ }^{4}$ UFMA (Universidade Federal do Maranhão)

${ }^{5}$ SEMED (Prefeitura Municipal de Matões do Norte, Maranhão)
} 


\section{RESUMEN}

El ensayo analiza el proceso de experimentar placer-estrés en la cultura organizacional. La calidad de vida nos lleva a la reflexión crítica y sistémica en medio del mundo laboral. Teniendo en cuenta que esta experiencia trae perjuicios para el empleado y para la organización (varios problemas de salud, absentismo y reducción de la productividad), pretendemos analizar las implicaciones de la cultura organizacional que favorecen el estrés en el empleado. Utilizamos como metodología el análisis de la literatura relevante al Síndrome de Burnout y las relaciones en el mundo laboral, así como los aspectos destacados por los autores sobre las formas de prevención y tratamiento del síndrome. Destacamos que los referentes teóricos que utilizamos son en las áreas de salud, administración y psicología. Finalmente, destacamos que el psicólogo es una figura central en el proceso de prevención y tratamiento del Síndrome de Burnout.

Palabras llave: Estrés; Cultura y clima organizacional; Síndrome de burnout; Psicología del trabajo y las organizaciones; Psicología.

\section{INTRODUÇÃO}

As organizações buscam profissionais qualificados capazes de bater metas estipuladas pelos seus dirigentes e produzir sempre mais, ficando a saúde e o bem-estar dos colaboradores em segundo plano, ou ainda, a qualidade de vida não passa de mero discurso. As pessoas são seres biopsicossociais e consequentemente muito complexas e com muitas necessidades, sendo o trabalho uma delas. É por meio do trabalho que a sociedade capitalista (especialmente a neoliberal) reconhece os indivíduos/sujeitos como cidadãos dignos e socialmente capazes.

O mundo do trabalho é cada vez mais competitivo, onde sobrevive quem possui maior empregabilidade. A empresa (organização) retém pessoas com capacidade de constante produtividade, flexibilidade, que buscam incessantemente a melhoria nas suas atividades. Isso vai para além dos conhecimentos técnicos. Atualmente, para sobreviver dentro das organizações, ter boas relações interpessoais com os colegas de trabalho e com o chefe, torna-se importante, - essencial.

Destacamos as vivências de prazer-sofrimento na organização do trabalho caracterizado

Pelo conteúdo da tarefa e relações socioprofissionais, a qual exerce um impacto no funcionamento psíquico do trabalhador gerando prazer-sofrimento 
dependendo do quanto a tarefa é significativa para o trabalhador e se as relações com colegas e chefias são ou não de reconhecimento, cooperação, confiança e solidariedade. (MENDES; TAMAYO, 2001, p. 40).

Campos e Costa (2007, p. 281) alertam que "O papel desempenhado pela cultura organizacional tem auxiliado o processo de formação e manutenção dos sentimentos que o indivíduo nutre por seu trabalho e pela organização". Embasados em Tamayo et al. (2004), as autoras destacam que:

Ao realizar o trabalho, sentimentos e emoções estão presentes no trabalhador, permeados pela cultura da organização: prazer de realizar o trabalho e angústia em executá-lo sem as condições necessárias, ansiedade por correr riscos, ocultar informações importantes, medo de ser responsável por resultados indesejados, ou mesmo responder com processo de adoecimento (depressão, LER/DORT, Burnout, etc.). (CAMPOS; COSTA, 2007, p. 281).

Como se pode observar acima, as exigências para produzir mais são constantes, o medo de perder o emprego acarretam doenças físicas, psicológicas e sociais, como: LER (lesões por esforços repetitivos) e DORT (distúrbios osteomusculares), estresse, irritação, dificuldades de concentração, falta de comunicação e depressão.

\footnotetext{
Por parte da medicina, que segue os pressupostos do modelo bio-médico, o diagnóstico "estresse" autoriza a receitar para o paciente uma série de medicamentos (ansiolíticos, anti-depressivos), que têm como único efeito, tranquilizá-lo, tirar a sua ansiedade, devolver o seu sono, retirar, enfim, todos os sintomas que apontam para complicações em sua saúde. Esses procedimentos são bastante graves, tanto em termos de saúde quanto em termos sociais. Eliminar sintomas podem mascarar o surgimento e o aparecimento de doenças tanto físicas quanto psíquicas - problemas hormonais, coronários, de obesidade e anorexia, psicose depressiva, comportamentos obsessivos, dependência química, dentre outros.

Por outro lado, as ações das ciências médicas, que separam o sujeito do ambiente em que vive, reforçam e reproduzem a opressão do capital sobre os corpos e as mentes dos seres humanos, em prol da maximização da mais-valia. Tais ações fazem, ainda, crescer de modo expressivo a indústria farmacêutica e o consumo de medicamentos. (LAGES, 2008, p. 6).
}

De acordo com o que traz Lages (2008), infere-se que em vez de buscar estratégias de bem-estar por outras vias como a psicológica, utiliza-se, com muita frequência, o modelo biomédico, onde o remédio é tido como aquele que tudo resolve. No entanto, as organizações possuem recursos para melhorar o ambiente de trabalho, minimizando esses conflitos e possíveis afastamentos por doenças advindas dessas exigências. Mas, muitas empresas vêm os cuidados como custos e não como investimento em qualidade, seja de vida, seja do trabalho e uma consequente produtividade. Pêgo e Pêgo (2016, p. 171) destacam que: “A síndrome necessita ser reconhecida como doença, para que possam ser 
implementadas medidas para sua prevenção”. Segundo Dalcin e Carlotto (2018, p. 141) "A Síndrome de Burnout é caracterizada como um estresse crônico que impacta o trabalhador na sua saúde mental e física e requer ações de prevenção e intervenção". De acordo com Cândido e Souza (2017) a Síndrome de Burnout é vista como um problema social que cada vez mais tem ganhado destaque. As autoras analisam as causas, os sintomas e a relação da SB com a tecnologia nas relações disfuncionais de trabalho, apontando tratamentos disponíveis atualmente.

É necessário pensar em outras maneiras de fazer gestão que possa ouvir e respeitar as opiniões e trabalhar o bem-estar dos colaboradores, promover cursos e palestras sobre relações interpessoais e harmonia no ambiente organizacional, e desenvolvendo a comunicação entre as pessoas que fazem e mantém a empresa como a melhor no mercado. E, cujos colaboradores venham favorecer com o que for necessário para criar e manter um ambiente favorável, harmonioso durante a jornada de trabalho, favorecendo a saúde de modo geral na organização. Visando qualificação e se mantendo informado sobre o que acontece no mundo do trabalho.

Neste artigo discutiremos o estresse e cultura e clima organizacional, destacando o papel do profissional da psicologia na prevenção e no tratamento da Síndrome de Burnout. Segundo Pêgo e Pêgo (2016, p. 171), “A Síndrome de Burnout (SB) assume uma concepção multidimensional, cuja manifestação se caracteriza por esgotamento emocional, redução da realização pessoal no trabalho e despersonalização do profissional".

Destacamos a atualização deste estudo em Pêgo e Pêgo (2016) que realizou revisão da literatura em língua portuguesa, sobre a Síndrome de Burnout nas principais bases de dados, como: Scientific Electronic Library Online (SciELO); Literatura LatinoAmericana e do Caribe em Ciências da Saúde (LILACS); National Library of Medicine (Medline), do Centro Latino-Americano e do Caribe de Informação em Ciências da Saúde (BIREME), entre 2002 e 2014, conferiram que é escasso o entendimento sobre a necessidade de considerar a SB como questão de saúde pública, destacando também que a literatura brasileira sobre SB é escassa quando comparada à internacional. "A partir do presente estudo, pode-se concluir que o Burnout tem efeitos negativos para a organização, para o indivíduo e para sua profissão. Conforme levantamento bibliográfico, a SB é um grave problema de saúde pública”. (PÊGO; PÊGO, 2016, p. 175). 
Uma pesquisa que nos chamou a atenção foi a de Cardoso et al. (2017) em que os autores destacam o início dos estudos sobre a Síndrome de Burnout no Brasil em meados de 1970. Os autores realizaram busca por artigos empíricos nas bases Scielo e PepSIC entre os anos de 2006 a 2015.

\section{A SAÚde DO TRABALHADOR FRENTE AOS PROCESSOS DE REESTRUTURAÇÃO PRODUTIVA}

O toyotismo é um entrave à promoção da saúde, tanto dos indivíduos quanto das comunidades. A gestão da subjetividade é prejudicada quando o indivíduo adere aos valores das organizações. A saúde do trabalhador toma evidência a partir de dois momentos da história do mundo do trabalho: do fordismo em 1913 e o toyotismo em 1945, aquele inicia a produção em série, aperfeiçoando a administração científica seguindo a trilha deixada por Taylor, este reestrutura a produção, que é rapidamente difundida pelo mundo, provocando profundas transformações na organização do trabalho e no perfil do trabalhador. (ANTUNES, 2002; LAGES, 2008).

O fordismo/toyotismo está voltado para a produção e a gestão baseados em um sistema de organizações técnicas e organizacionais com o objetivo de elevar a produção, o consumo de massa e a consequente acumulação de capital.

\section{TRABALHO E CULTURAL ORGANIZACIONAL}

Para Tamayo et al. (2004), há uma estreita relação entre a composição do ser humano, o processo de civilização e a produção do trabalho, os quais podem ser chamados de marcas da cultura. É sabido que o trabalho é considerado um dos fundamentos da cultura humana, provavelmente um dos mais inerentes a ela, gerando bens, riquezas, e infelizmente, também, danos à saúde das pessoas. A organização do trabalho deveria garantir às pessoas prazer, satisfação pessoal e profissional, e sua permanente pretensão à felicidade.

No entanto, o homem devota grande parte de sua vida ao trabalho e o surgimento de doenças está amplamente relacionado aos impactos da organização do trabalho sobre a saúde, o bem-estar físico, social e mental dos trabalhadores. A definição de saúde organizacional está expressamente associada à harmonia entre o bem-estar do indivíduo, a estrutura, e o funcionamento organizacional. 
A cultura é a essência de uma organização e, provavelmente, um de seus fundamentos. Sabe-se que as organizações são um aglomerado de pessoas em torno do trabalho para produzir riquezas, bens e mercadorias. A cultura esta relacionada ao modo de produção social do trabalho, é um elemento abrangente, universal e intangível na organização e pode ser claramente observado no modo das pessoas se vestirem, se comunicarem, se organizarem, como criam ferramentas de manuseio de objetos humanos no trabalho.

A cultura é muito saudável quando reforça o prazer e a realização do trabalhador, é prejudicial quando nega a identidade e a realização profissional e pessoal do colaborador e produz sofrimento, sobrecargas físicas e mentais profundamente relacionadas aos agravos à saúde, relacionados ao acúmulo de trabalho, stress, esgotamento profissional ou Burnout, depressão e distúrbios psicossomáticos. O entendimento da cultura organizacional beneficia o conhecimento de como vivem e por que os trabalhadores adoecem.

Tamayo et al. (2004), afirma que, em uma perspectiva histórica, o trabalho humano revela sua evolução num meio civilizado e cultural. Além disso, evidencia para as pessoas uma das características mais significativas de sua vida e inerente à sua existência. Não é apenas a produção de riquezas e bens, mas também a forma de sua realização como indivíduo.

A dependência entre o trabalho e o bem-estar do colaborador necessita que as organizações implantem e implementem uma política de prevenção, criando uma cultura organizacional, em que seja propícia a favorável execução da função da organização com a garantia da qualidade de vida e realização pessoal.

De acordo com Mendes e Tamayo (2001) os valores da organização constituem um dos importantes instrumentos para compreender a cultura organizacional. Tendo o papel de atender tanto aos objetivos organizacionais, quanto de atender às necessidades dos indivíduos, conciliando-os para a possível sobrevivência da organização.

Considerando esse modelo,

[...] os valores não estão definidos a priori e podem ser classificados numa hierarquia, não são fixos, variam de uma organização para outra e até dentro da própria organização. Os valores organizacionais servem à própria sobrevivência da organização e, por essa razão, buscam mediar conflitos para resolver problemas. (MENDES; TAMAYO, 2001, p. 40). 
O pressuposto é de que é a cultura que permite a liberdade dos indivíduos em aceitar ou não determinado conteúdo ou a eficiência do controle dessa liberdade.

\section{AS VIVÊNCIAS DE PRAZER-SOFRIMENTO NAS ORGANIZAÇÕES}

Destacamos os estudos de Tamayo et al. (2004 apud CAMPOS; COSTA, 2007) que traz a importância da cultura organizacional como indicador de eficiência da organização na valorização dos seus recursos humanos. Ferreira e Assmar (apud CAMPOS; COSTA, 2007) dizem de uma relação de poder e dominação que uma organização que implica os processos de saúde no trabalho.

As vivências de prazer-sofrimento na organização do trabalho se caracteriza pelo conteúdo de fazeres e tarefas assim como as relações socioprofissionais que exercem impacto no funcionamento psíquico do trabalhador, o que pode gerar prazer-sofrimento, a depender do significado que o trabalhador dar à tarefa. Outro fator importante para caracterizar essa dúbia relação do trabalhador com o ambiente de trabalho, o que implica no sentido do prazer-sofrimento, são as relações que mantém com colegas e chefias, se são de reconhecimento ou não, e ainda, se de cooperação, confiança e solidariedade. (MENDES; TAMAYO, 2001).

Nesse sentido, os valores organizacionais como norteadores das atitudes dos colaboradores, proporcionam prazer quando existe uma organização do trabalho flexível ou negociável. Caso contrário, poderá fomentar o sofrimento no trabalho. Mendes e Tamayo (2001) apontam como características da vivência de prazer-sofrimento, a valorização e o reconhecimento que definem o prazer e o desgaste com o trabalho. Ao mesmo tempo em que o colaborador tem seu trabalho valorizado em discurso, gerando prazer, há o desgaste devido ao descontentamento com o trabalho.

É preciso que sejam observados os valores organizacionais a fim de interferir nas vivências de prazer-sofrimento, buscando contradições, valores que não estão presentes à realidade da organização, entre outros. Segundo Campos e Costa (2007, p. 281) "O papel desempenhado pela cultura organizacional tem auxiliado o processo de formação e manutenção dos sentimentos que o indivíduo nutre por seu trabalho e pela organização". Cultura esta, que na vivência de prazer-sofrimento é caracterizada por relações conflitantes, de competição e metas exorbitantes. Além disso, deve ser levado em 
consideração aspectos pessoais, como: renda familiar, a satisfação e as necessidades básicas do colaborador.

Tem por consequência deste processo: problemas de saúde que interferem na rotatividade, no absenteísmo e na produtividade da organização, acarretando perda de bons profissionais, despesas adicionais com seleção e recrutamento, e outros desdobramentos na eficácia da organização (CAMPOS; COSTA, 2007).

\section{O ESTRESSE NAS ORGANIZAÇÕES}

As organizações buscam perfis detalhados para seus colaboradores, este deve estar em constante desenvolvimento intelectual, ser flexível, ter competências especificas, ser engajado entre outras características. Engajado pode ser facilmente relacionado ao tema estresse, pois, estar "estressado" pode trazer para alguns indivíduos, sentimentos de importância, de reconhecimento dentro da empresa, principalmente em organizações que possuem ambientes altamente competitivos. Surge então nas organizações um novo termo, workaholics, pessoas que são viciadas no trabalho. "Os workaholics são muito valorizados no meio empresarial, pois são pessoas muito produtivas, competitivas, eficazes, vivem para a empresa e em busca de objetivos relacionados ao trabalho, com alto nível de rendimento profissional" (FRANÇA; RODRIGUES, $1999^{2}$ apud STEFANICZEN; STEFANO; MACHADO, 2010, p. 2).

É importante para a manutenção do "estresse positivo", que a definição de metas e prazos estejam de acordo com a capacidade e disponibilidade de cada empregado. É exatamente esse nível de exigência adequado que determina um bom rendimento. Abaixo da linha do estresse positivo, o rendimento diminui bastante. Na via oposta, prazos irreais e demandas excessivas compõem um cenário desajustado, que favorece a manifestação dos sinais negativos do estresse.

As pessoas reagem de diversos modos às situações e aos fatores ambientais que provocam estresse, a tolerância para a ambiguidade, paciência, autoestima, saúde e exercícios físicos e hábitos de trabalho e de sono afetam a maneira como elas reagem ao

\footnotetext{
${ }^{2}$ LIMONGI-FRANÇA, Ana Cristina; RODRIGUES, Avelino Luiz. Stress e trabalho: uma abordagem psicossomática. [S.l: s.n.], 2002.
} 
estresse. Genuíno, Gomes e Moraes (2010) com base em Chiavenato (2008) apontam dois fatores principais do estresse no trabalho: ambiental e pessoal.

\begin{abstract}
Cada pessoa reage sob diferentes maneiras na mesma situação aos fatores ambientais que provocam estresse. Pessoas que são viciadas no trabalho (workaholics) e que são impulsionadas para alcançar metas - geralmente estão mais sujeitas ao estresse do que as outras. Sua tolerância para a ambigüidade, paciência, auto-estima, saúde e exercícios físicos e hábitos de trabalho e de sono afetam a maneira como elas reagem ao estresse. O estresse não é necessariamente disfuncional. Algumas pessoas trabalham bem sob pequena pressão e são mais produtivas em uma abordagem de cobrança de metas. Outras buscam incessantemente mais produtividade ou um melhor trabalho. Um nível modesto de estresse conduz a maior criatividade quando uma situação competitiva conduz a novas idéias e soluções. (GENUÍNO; GOMES; MORAES, 2010, p. 5).
\end{abstract}

Porém as organizações devem ter extrema atenção ao dosar essa pressão em seus colaboradores, levando sempre em consideração a subjetividade de seus empregados, quando realizado de forma sadia e satisfatória, o trabalho promove reconhecimento social, identidade e autoestima ao trabalhador, e acarreta aumento da produtividade e qualidade do trabalho.

Contudo, quando os níveis de ansiedade, tensão, competitividade e perfeccionismo são altos, o risco de estresse e Burnout são consideráveis.

Os profissionais que sofrem pressões diariamente no local de trabalho, correm o risco de adquirir a Síndrome de Burnout, um esgotamento físico e mental que traz uma série de sintomas para a vida profissional e pessoal do indivíduo. "As causas e os sintomas não são universais. Dependendo das características da pessoa e das circunstâncias em que esta se encontre, o grau e as manifestações são diferentes". (BENEVIDES-PEREIRA, 2001, p. 34 apud SHIRAISHI, 2014).

Benevides-Pereira (2001), mostra um quadro de quatro categorias de sintomas do Burnout. Físicos: fadiga constante e progressiva, distúrbios do sono; comportamentais: negligência ou excesso de escrúpulos, irritabilidade; psíquicos: falta de atenção, de concentração, alterações de memória; defensivos: sentimento de onipotência, absenteísmo. [adaptado, grifo nosso]. O Portal da Educação, aponta que quando a Síndrome de Burnout já estiver instalada, o tratamento pode ser realizado por meios físicos, psíquicos e sociais: "exercícios físicos regulares; - repouso, lazer e diversão."; [...] "busca de convivência menos conflituosa com pares e grupos."; [...] revisão e redimensionamento das formas de organizações de trabalho; [...] (GIL-MONTE, 2003 apud PORTAL DA EDUCAÇÃO, 2020). 
Transformações que marcam os sistemas de gestão contemporâneos sobre as relações sujeito-trabalho-organização, há de se salientar, entre outros aspectos, o fato de o trabalhador passar a viver a insegurança de obter trabalho e rendimento suficientes para sua manutenção, sendo levado a gerir-se como dono de sua própria carreira (SANT'ANA; CARVALHO; FONSECA, 2006, p. 9).

O Portal da Educação, na aba Psicologia, traz Gil-Monte (2003) e aponta que “[...] as estratégias de prevenção e tratamento do burnout podem ser agrupadas em três categorias: individuais, grupais e organizacionais.”.

\begin{abstract}
A) Estratégias individuais: referem-se à formação em resolução de problemas, assertividade, e gestão do tempo de maneira eficaz; B) Estratégias grupais: consistem em buscar o apoio dos colegas e supervisores. Deste modo, os indivíduos melhoram as suas capacidades, obtêm novas informações e apoio emocional, ou outro tipo de ajuda; C) Estratégias organizacionais: muito importantes porque o problema está no contexto laboral, consistem no desenvolvimento de medidas de prevenção para melhorar o clima organizacional, tais como programas de socialização para prevenir o choque com a realidade e implantação de sistemas de avaliação que concedam aos profissionais um papel ativo e de participação nas decisões laborais. (PORTAL DA EDUCAÇÃO, 2020)
\end{abstract}

A gestão das organizações normalmente relega a um segundo plano a necessidade de um ambiente saudável, classificando o mal-estar como um problema exclusivo do colaborador. No entanto, empresas mais desenvolvidas já corrigem esse viés, pois sabem que a performance do funcionário afeta diretamente os resultados corporativos e a competência dos colaboradores passa a ser reconhecida como o ativo principal da empresa.

\title{
O PAPEL DO PSICÓlOGO NA PREVENÇÃO E TRATAMENTO DA SÍNDROME DE BURNOUT
}

É importante que nas empresas, as áreas de saúde e segurança trabalhem de forma multidisciplinar com o psicólogo procurando desenvolver programas e atividades focados nesse tema, atuando de forma preventiva com os trabalhadores que a partir de algumas características estejam mais suscetíveis à Síndrome de Burnout.

Portanto, é essencial que se cuide melhor da saúde física e mental, alimentandose e dormindo-se bem, exercitando-se regularmente, além de possuir vida social ativa. Algumas atividades sugeridas como palestras de alerta ao tema, dinâmicas de grupo que tenha como foco tirar aquele funcionário da rotina, ter por algumas horas uma distração, 
uma diversão, podem evitar estresse e consequentemente, sintomas que causadores do Burnout.

\begin{abstract}
Os métodos para tratamento e prevenção do burnout podem ser caracterizados em três categorias: Individual, grupal e organizacional ${ }^{3}$.

As estratégias individuais referem-se à formação em resolução de problemas, assertividade, e gestão do tempo de maneira eficaz.

As estratégias grupais consistem em buscar o apoio dos colegas e supervisores. Deste modo, os indivíduos melhoram as suas capacidades, obtêm novas informações e apoio emocional ou outro tipo de ajuda.

Por fim, as estratégias organizacionais, muito importantes porque o problema está no contexto laboral, consistem no desenvolvimento de medidas de prevenção para melhorar o clima organizacional. As estratégias mais relevantes para a prevenção da síndrome de burnout no trabalho que pode ser implantado desde a direção da organização, são os primeiros programas de socialização, a implementação de sistemas avaliação e feedback e processos de desenvolvimento organizacional. (GIL-MONTE, 2003, p. 30-31, tradução nossa).
\end{abstract}

O tratamento para a Síndrome de Burnout consiste na união de psicoterapia; atividades de relaxamento; e dependendo da gravidade, até mesmo o uso de medicamentos como antidepressivos ou tranquilizantes, no entanto, uma mudança drástica na dinâmica diária e estilo de vida, se faz necessária para reforçar estes tratamentos.

Para colaboradores que já foram identificados com a Síndrome, é preciso ter um nível maior de atenção. O gestor imediato deve ser chamado para contribuir no tratamento, pois é necessário que a rotina desse funcionário passe por alterações, como redução de atividades ou redução da carga horária do trabalho. Incluir também em sua rotina atividades diferenciadas, incentivando a participar nos programas externos se a

\footnotetext{
${ }^{3}$ Las estrategias de intervención para la prevención y tratamiento del síndrome de quemarse por el trabajo pueden ser agrupadas en tres categorías: estrategias individuales, estrategias grupales y estrategias organizacionales (Gil-Monte y Peiró, 1997).

Dentro de las estrategias del nivel individual se recomienda la utilización del entrenamiento en solución de problemas, el entrenamiento de la asertividad, y los programas de entrenamiento para manejar el tiempo de manera eficaz. En el nivel grupal la estrategia por excelencia es la utilización del apoyo social en el trabajo por parte de los compañeros y supervisores. A través del apoyo social en el trabajo los individuos obtienen nueva información, adquieren nuevas habilidades o mejorar las que ya poseen, obtienen refuerzo social y retroinformación sobre la ejecución de las tareas, y consiguen apoyo emocional, consejos, u otros tipos de ayuda. Por último, es muy importante considerar el nivel organizacional, pues el origen del problema está en el contexto laboral y, por tanto, la dirección de la organización debe desarrollar programas de prevención dirigidos a mejorar el ambiente y el clima de la organización. Las estrategias más relevantes para la prevención del síndrome de quemarse por el trabajo que pueden ser implantadas desde la dirección de la organización son los programas de socialización anticipada, la implantación de sistemas de evaluación y retroinformación, y los procesos de desarrollo organizacional.
} 
empresa possuir como: trabalho voluntário na comunidade, gincanas internas, passeios proporcionados para empregados.

\begin{abstract}
Destaca-se a maior relevância de maiores conversações (CLEGG e HARDY, 1999) entre a administração e outros campos do saber, entre elas a psicanálise, que, em sua extensão, apresenta um potencial significativo a ser explorado, de forma mais profunda, quanto a novos olhares e abordagens teóricometodológico conceituais, capazes de propiciar visões mais abrangentes acerca das múltiplas facetas que caracterizam a complexidade das relações sujeitotrabalho-organização, na contemporaneidade (ÉNRIQUEZ, 1997; CHANLAT, 1996; AKTOUF, 1996; LAPIERRE, 1995; PAGÉS et al., 1987) (SANT'ANA; CARVALHO; FONSECA, 2006, p. 14).
\end{abstract}

Portanto, destaca-se que o tratamento com psicólogo é fundamental para aqueles que já foram acometidos pela Síndrome. O apoio do profissional de Psicologia se mostra de extrema importância no tratamento do Burnout, mas não substituindo nenhum programa implementado pelas equipes multidisciplinares.

\title{
CONSIDERAÇÕES FINAIS
}

As discussões feitas nesse ensaio evidenciam a complexidade que envolve o ambiente de trabalho e a relação estabelecida com o trabalhador, sujeito pensante e portador de um organismo biopsicossocial que age em constante interação com o meio, modificando-o e sendo modificado, demandando mais pesquisas e projetos que ampliem a compreensão desta realidade e proponha alternativas práticas de melhoria na organização e consequentemente na qualidade de vida do trabalhador.

Os autores deste ensaio iniciam a partir da constatação de que o mundo do trabalho está cada vez mais exigente e, se comparado à preocupação que as empresas possuem quanto às metas e os lucros, o que tange à saúde do colaborador pouco está sendo feito diante da vasta gama de consequentes que podem ser desencadeados caso esse funcionário esteja inserido em um ambiente organizacional patológico.

No entanto, o sujeito, por sua vez, se submete a essas condições, uma vez que é desse lugar que provê os recursos necessários para que a família, os planos e objetivos de vida se sustentem. Em paralelo, concordam com Tamayo et al. (2004) que o trabalho além de todas as conquistas materiais, permite a realização pessoal do indivíduo, constituindo assim uma relação de dependência entre o fazer e o bem-estar que pode ser oportunizado por meio de políticas de prevenção e de uma cultura organizacional capaz de propiciar a satisfação tanto para empregados quanto empregadores. 
Devendo-se ressaltar, todavia, que o desenvolvimento de análises, como essa, torna-se justificável tanto pela compreensão que se tem a cerca dos danos biopsicossociais que as condições do ambiente de trabalho influenciam na vida do trabalhador, quanto pelo reconhecimento da relevância de uma cultura organizacional como indicador de eficiência da organização na valorização dos seus recursos humanos. Sendo pertinente ainda, observar, como dito por Mendes e Tamayo (2001), que o conteúdo da tarefa e das relações socioprofissionais exerce um impacto no funcionamento psíquico do trabalhador gerando prazer-sofrimento dependendo do quanto a tarefa é significativa para o trabalhador e do quanto as relações com colegas e chefias são ou não de reconhecimento, cooperação, confiança e solidariedade.

Por fim, o grande desafio envolvido ao longo de todas as questões levantadas diz respeito à enorme variabilidade que existe de um ambiente para o outro e entre sujeitos, o que aumenta ainda mais a rede de micro e macro análises que podem ser feitas acerca dos reforçadores e estressores, assim como, da qualidade de vida do trabalhador, da cultura organizacional e das exigências impostas pelo mercado tanto para as empresas quanto para os empregadores que desejam se manter ativos. 


\section{REFERÊNCIAS}

ANTUNES, R. Os sentidos do Trabalho - ensaios sobre a afirmação e a negação do trabalho. São Paulo: Boitempo Editorial, 2002.

BENEVIDES-PEREIRA, A. M. T. A Saúde Mental dos Profissionais de Saúde Mental. Maringá: Eduem, 2001.

CÂNDIDO, Jéssica; SOUZA, Lindinalva Rocha de. Síndrome de Burnout: as novas formas de trabalho que adoecem. Disponível em:

http://ead.bauru.sp.gov.br/efront/www/content/lessons/82/BURNOUT\%20PSICOLOGI A.pdf. Acesso em: 21 dez. 2021.

CAMPOS, I. C. M.; COSTA, F. de N. Cultura e saúde nas organizações. Estud. psicol. (Campinas) [online]. 2007, v. 24, n. 2, p. 279-282. Disponível em: https://www.scielo.br/j/estpsi/a/KPcTvrVcB4rC7wbPVkRgmKN/?lang=pt. DOI https://doi.org/10.1590/S0103-166X2007000200014. Acesso em: 09 dez. 2021.

CARDOSO, Hugo Ferrari et al . Síndrome de burnout: análise da literatura nacional entre 2006 e 2015. Rev. Psicol., Organ. Trab., Brasília , v. 17, n. 2, p. 121-128, jun. 2017 . Disponível em <http://pepsic.bvsalud.org/scielo.php?script=sci_arttext\&pid=S1984$66572017000200007 \& \operatorname{lng}=$ pt\&nrm=iso $>$.

Acesso em: 22 dez. 2021. http://dx.doi.org/10.17652/rpot/2017.2.12796.

DALCIN, Larissa; CARLOTTO, Mary Sandra. Avaliação de efeito de uma intervenção para a Síndrome de Burnout em professores. Psicologia Escolar e Educacional [online]. 2018, v. 22, n. 1 [Acessado 22 dezembro 2021], pp. 141-150. Disponível em: <https://doi.org/10.1590/2175-35392018013718>. Epub Jan-Apr., 2018. ISSN 21753539. https://doi.org/10.1590/2175-35392018013718.

GIL-MONTE, P. El Síndrome de Quemarse por el Trabajo en Enfermería. Revista Eletrônica InterAção Psy. Ano 1, n. 1, 2003, 19-33. Disponível em: https://docplayer.com.br/19084534-Revista-eletronica-psy-ano-1-no-1.html. Acesso em: 09 dez. 2021.

GENUÍNO, S. L. V. P.; GOMES, M. da S.; MORAES, E. M. de. O Estresse Ocupacional e a Síndrome de Burnout no Ambiente de Trabalho: Suas Influências no Comportamento dos Professores da Rede Privada do Ensino Médio de João Pessoa. Anagrama, [S. l.], v. 3, n. 2, p. 1-9, 2009. Disponível em: https://www.revistas.usp.br/anagrama/article/view/35426. Acesso em: 9 dez. 2021.

LAGES, S. R. C. A saúde do trabalhador frente aos processos de reestruturação produtiva. Estação Científica Online (Ed. Esp. Saúde) Juiz de Fora, n. 05, jan. 2008. Disponível em: https://portaladm.estacio.br/media/4322/8-a-saude-trabalhador-frente-processosreestruturacao-produtiva.pdf. Acesso em: 09 dez. 2021.

MENDES, A. M.; TAMAYO, A. Valores organizacionais e prazer-sofrimento no trabalho. Psico - USF, v.6, n.1, p.39-46, jan./jun. 2001. Disponível em: https://www.scielo.br/j/pusf/a/RM8VSqwSqqSYzgC7c8y7gBg/?format=pdf\&lang=pt. Acesso em: 09 dez. 2021. 
PÊGO, F. P. L. e; PÊGO, D. R. Síndrome de Burnout. Rev Bras Med Trab. 2016, v. 14, n. 2, p. 171-6. Disponível em: https://cdn.publisher.gn1.link/rbmt.org.br/pdf/v14n2a15.pdf. Acesso em: 09 dez. 2021.

Prevenção e Tratamento do Burnout. Portal da Educação. Psicologia. 2020. Disponível em: https://siteantigo.portaleducacao.com.br/conteudo/artigos/psicologia/prevencao-etratamento-do-burnout/28225. Acesso em: 09 dez. 2021.

SANT'ANA, A. de S.; CARVAlHO, R. A. A. de; FONSECA, S. T. D. Contemporaneidade e novas formas de relação sujeito-trabalho-organização: o que nos tem a dizer a psicanálise? Cadernos de ideias. Ano 6, n. 30 - dez. 2006. Disponível em: https://ci.fdc.org.br/AcervoDigital/Cadernos\%20de\%20Id\%C3\%A9ias/2006/0630.pdf? Mobile $=1 \&$ Source $=\% 2 \mathrm{~F} \% 5$ Flayouts $\% 2 \mathrm{~F} 15 \% 2 \mathrm{Fmobile} \% 2 \mathrm{Fviewa} \% 2 \mathrm{Easpx} \% 3 \mathrm{FList} \% 3$ D6beaddb7\%2D4351\%2D494c\%2D9d58\%2D6dc358918be9\%26View\%3Dbf7ddbda\% 2D36c4\%2D452b\%2D95db\%2D51b60f87de4e\%26RootFolder\%3D\%252FAcervoDigit al\%252FCadernos \%2Bde\%2BId\%25u00e9ias\%252F2006\%26PageFirstRow\%3D21\%2 6wdFCCState\%3D1. Acesso em: 09 dez. 2021.

SHIRAISHI, J. B. T. Síndrome de Burnout - Síndrome de Burnout é um distúrbio psíquico de caráter depressivo, precedido de esgotamento físico e mental intenso. 23 de outubro de 2014. Disponível em: https://administradores.com.br/artigos/sindrome-deburnout. Acesso em: 09 dez. 2021.

STEFANICZEN, J.; STEFANO, S. R.; MACHADO, R. de O. WORKAHOLIC: um novo modelo de comportamento nas organizações. In: XIII SemeAd: Seminários em Administração. Setembro de 2010, ISSN 2177-3866. Disponível em: http://www.sgc.goias.gov.br/upload/arquivos/2013-04/area-tematica--gestao-depessoas-workaholic-um-novo-modelo-de-comportamento-nas-organizacoes.pdf. Acesso em: 09 dez. 2021.

TAMAYO, A. et al. (Orgs.). Cultura e saúde nas organizações. São Paulo: Artmed. Livros, 2004. 